\section{Successful Management of Postoperative Pancreatic Fistula by Application of Constructed S-Type Pancreatic Stent After Operation for Abnormal Biliary-Pancreatic Junction}

We describe here our experience of successful closure of a lesion of the main pancreatic duct by means of a constructed S-type pancreatic stent.

A 67-year-old woman with an abnormal biliary-pancreatic junction and a tumor of the gallbladder underwent cholecystectomy plus R2, extrahepatic cholangiectomy, hepatocholangiojejunostomy, and Roux-en-Y reconstruction on 2 February 2001. In the postoperative period pancreatic fistulization occurred, with endoscopic retrograde cholangiopancreatography (ERCP) revealing a leak of contrast medium medially to the common bile duct. A pancreatic stent (GEPD, 7-Fr; Wilson-Cook, Winston-Salem, North Carolina, USA) was therefore put in place. Unfortunately, the stent migrated 2 days later, and so it was replaced with a constructed pancreatic stent with a shape which approximated to that of the main pancreatic duct (Figure 1) (constructed Stype pancreatic stent, 7-Fr; Olympus, Tokyo, Japan). This was followed by excellent progress, which allowed removal of the stent and discharge of the patient from hospital 6 weeks later.

In our department, we have been using straight biliary stents (PBD, Olympus; or GEPD, Wilson-Cook). However, as the main pancreatic duct has characteristic

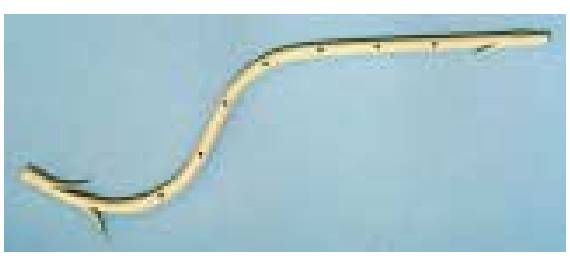

Figure 1 A constructed 7-Fr stent (Olympus) with a shape approximating that of the main pancreatic duct. See text for further description. bends within the pancreatic head, we were not able to stabilize the fixation of stents and consequently they migrated. Because of this, in 1998 we constructed a stent with a shape which approximated to that of the main pancreatic duct. The tip of the stent tapers and sideholes have been added. The two bends in the stent correspond to the shape of the main pancreatic duct. The material used is a polyolefin-based elastomer, with a flexibility which is $50-70 \%$ greater than that of PBD; this is important for the adaptation of the stent to interindividual variability in the shape of the main pancreatic duct (Figure 2). We applied the stent in the case of a patient with chronic pancreatitis, with excellent results [1-3]. Unfortunately, because of the migration of a GEPD stent the patient had become febrile, so we replaced it with an S-type stent and this resulted in remission. The stent closed the lesion of the main pancreatic duct, protected the flow through the duct, and also stayed in place. Based on these outcomes we concluded that the constructed S-type pancreatic stent was effective in the treatment of postoperative pancreatic fistula.

\section{T. Ukita ${ }^{1}$, A. Moriyama ${ }^{1}$, A. Tada ${ }^{1}$, J. Shimura ${ }^{1}$, H. Inoue ${ }^{1}$, T. Kakemura ${ }^{1}$, I. Maetani ${ }^{1}$, Y. Igarashi ${ }^{1}$, Y. Sakai ${ }^{2}$, M. Nakamura ${ }^{3}$}

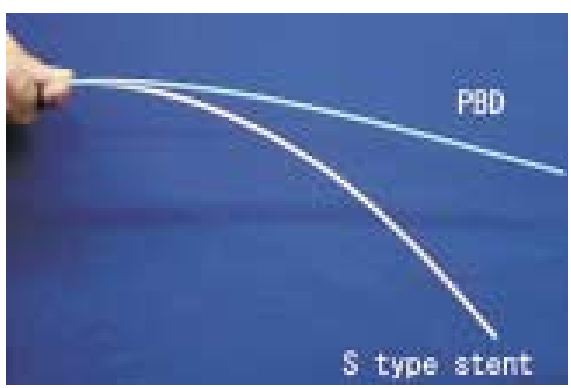

Figure 2 PBD (upper) and constructed (lower) stent material.
${ }^{1}$ Third Department of Internal Medicine, Toho University School of Medicine,

Tokyo, Japan

${ }^{2}$ Division of Digestive Endoscopy, Toho University Ohashi Hospital, Tokyo, Japan

${ }^{3}$ Third Department of Surgery, Toho University School of Medicine, Tokyo, Japan

\section{References}

${ }^{1}$ Ukita T, Igarashi Y. Studies on endoscopic pancreatic stents for acute pancreatitis and chronic pancreatitis. Progr Dig Endosc 1999; 54: 148

${ }^{2}$ Igarashi Y, Ikeda M, Tada T et al. Endoscopic pancreatic drainage treatment of acute and chronic pancreatitis [in Japanese]. Rinsho Shokakinaika (Clinical Gastroenterology) 2000; 15: 665-671

${ }^{3}$ Ukita T, Maetani I, Igarashi Y. Studies of S-type pancreatic stent constructed for prevention of migration. Progr Dig Endosc 2001; 60: 51

\section{Corresponding Author}

\section{T. Ukita, M.D.}

Third Department of Internal Medicine Toho University School of Medicine

Meguro-ku

Ohashi 2-17-6

Tokyo 153-8515

Japan

Fax: $\quad+81-3-34681269$

E-mail: uki@oha.toho-u.ac.jp 